\title{
Leigh syndrome T8993C mitochondrial DNA mutation: Heteroplasmy and the first clinical presentation in a Vietnamese family
}

\author{
CHAMARA ARACHCHIGHE LAHIRU WEERASINGHE ${ }^{1}$, BICH-HONG THI BUI ${ }^{1}$, THU THI VU ${ }^{1}$, \\ HONG-LOAN THI NGUYEN ${ }^{1}$, BAO-KHANH PHUNG ${ }^{1}$, VAN-MINH NGUYEN ${ }^{1}$, \\ VAN-ANH PHAM ${ }^{2}$, VU-HUNG CAO ${ }^{2}$ and TUAN-NGHIA PHAN ${ }^{1}$ \\ ${ }^{1}$ Key Laboratory of Enzyme and Protein Technology, VNU University of Science; \\ ${ }^{2}$ Department of Neurology, Vietnam National Children's Hospital, Hanoi 100000, Vietnam
}

Received October 2, 2017; Accepted February 27, 2018

DOI: $10.3892 / \mathrm{mmr} .2018 .8670$

\begin{abstract}
Leigh syndrome is a rare inherited, heterogeneous and progressive neurometabolic disorder that is mainly caused by specific mutations in nuclear DNA (nDNA) or mitochondrial DNA (mtDNA). The present study reported a case of childhood Leigh syndrome with a point mutation at bp 8,993 in the mitochondrial ATPase6 gene. A 21-month-old male child had developed epilepsy, muscular weakness and vomiting, which was accompanied by high fever. Magnetic resonance imaging indicated typical characteristics of Leigh syndrome, including a symmetric abnormal signal in the dorsal medulla oblongata and Sylvian fissure enlargement in association with an abnormal signal in the periventricular white matter and in the putamina and caudate heads. The diagnosis was further supported with genetic tests including polymerase chain reaction-restriction fragment length polymorphism (PCR-RFLP), sequencing, and quantitative PCR. The patient was found to carry a mitochondrial T8993C (m.T8993C) mutation in peripheral blood with $94.00 \pm 1.34 \%$ heteroplasmy. Eight of his relatives were also subjected to quantification of the m.T8993C mutation. The percentages of heteroplasmy in samples taken from the grandmother, mother, aunt, cousin 1 , and cousin 2 were $16.33 \pm 1.67$, $66.81 \pm 0.85,71.66 \pm 3.22,87.00 \pm 1.79$, and $91.24 \pm 2.50 \%$, respectively. The mutation was not found in samples taken from the father, the husband of the aunt, or the grandfather of the patient. The obtained data showed that the mutation was maternally inherited and accumulated through generations. Even though the heteroplasmy levels of his mother, aunt, cousin 1 , and cousin 2
\end{abstract}

Correspondence to: Professor Tuan-Nghia Phan, Key Laboratory of Enzyme and Protein Technology, VNU University of Science, 334 Nguyen Trai Street, Thanhxuan, Hanoi 100000, Vietnam E-mail:phantuannghia@vnu.edu.vn

Key words: Leigh syndrome, mtDNA mutation, heteroplasmy, T8993C, polymerase chain reaction-restriction fragment length polymorphism, quantitative PCR were relatively high (66.81-91.24\%), they remained asymptomatic, indicating that the threshold at which this mutation shows effects is high. To the best of our knowledge, this is the first report of a case of Leigh syndrome in a Vietnamese individual harboring a mtDNA mutation at the $8,993 \mathrm{bp}$ site, and showing a correlation between the heteroplasmy and clinical phenotype. These findings may be useful in helping to improve the clinical diagnosis and treatment of Leigh syndrome.

\section{Introduction}

Leigh syndrome (also termed subacute necrotizing encephalomyelopathy) is a rare, inherited, heterogeneous, and progressive neurometabolic disorder (1). This syndrome is characterized by a wide variety of neurologic abnormalities $(2,3)$ with degeneration of cognitive and motor functions, in most cases resulting in death due to respiratory failure (4). Leigh syndrome normally begins between the ages of three months and two years, and affects approximately 1 in 40,000 live births worldwide (5). Neurological symptoms are hallmarks of Leigh syndrome and patients generally manifest a progressive decline in central nervous system function because of necrotizing lesions of the basal ganglia, cerebellum, or brainstem, as visualized by magnetic resonance imaging (MRI) (3). Additional clinical manifestations and ages of onset vary from case to case, encompassing a diverse array $(6,7)$. Leigh syndrome can result from specific mutations in nuclear DNA (nDNA) or mitochondrial DNA (mtDNA). Mutations in mtDNA are responsible for approximately $20 \%$ of cases of Leigh syndrome $(8,9)$. The main mutations, which are both associated with the ATPase6 gene, are m.T8993G and m.T8993C (10). They result in the most frequent cases of maternally inherited Leigh syndrome (MILS) $(4,10)$. The m.T8993C variant results in later onset, slower progression, and lesser severity than the energy production-related m.T8993G mutation $(9,11)$. The T8993G or T8993C mutations convert a leucine residue in the mitochondrial ATP synthase protein into arginine or proline, respectively, reducing its activity, and subsequently leading to ATP deficiency in the cells. T8993C-induced reduction of ATP synthesis is also involved 
with deficiency of complexes $\mathrm{I}$ and $\mathrm{V}$ in the mitochondrial oxidative phosphorylation system (OXPHOS) $(11,12)$, with consequent cell death. Moreover, experimental data previously revealed that the m.T8993C mutation leads to increased reactive oxygen species (ROS) production, thus creating oxidative stress (13). Therefore, high-energy demanding tissues, such as brain and heart, are most vulnerable to this process, which in part explains the clinical features of Leigh syndrome (4). The clinical manifestations of these gene defects largely depend on the abundance of mutated mtDNA molecules (12). High mutation loads (over 90\%) usually cause Leigh syndrome cases such as MILS, while lower levels can cause less severe phenotypes such as neuropathy, ataxia, and retinitis pigmentosa (NARP). Thus, a diverse array of clinical features $(10,14)$ presents a challenge to the clinicians (7). Therefore, for better diagnosis and treatment of this syndrome, molecular genetic testing is required, in addition to clinical characterization.

\section{Case report}

Samples. Peripheral blood samples were collected from a patient showing characteristics of Leigh syndrome and eight of his family members at the Vietnam National Children's Hospital (VNCH; Hanoi, Vietnam). The present study was approved by the ethics committee of the $\mathrm{VNCH}$ and conformed to the guidelines and ethical rules of the $\mathrm{VNCH}$. All the subjects signed a consent form for voluntary participation in the research.

Clinical description of patient. The patient was a 21-month-old male displaying characteristics of Leigh syndrome (movement disorder, hypotonia, fever, intractable nausea and vomiting, and abnormal brain MRI as shown in Fig. 1). Blood test results of the patient showed lactatemia $(8.2 \mathrm{mmol} / \mathrm{l})$ and acidosis associated with ketonuria. He was born after 40 weeks of gestation to a 32-year-old mother and a 35-year-old father via normal delivery. Only the patient showed clinical characteristics of Leigh syndrome whereas the other eight members of his family were normal. The patient died at 34 months of age.

MRI. Axial fluid-attenuated inversion recovery MRI (Axial FLAIR MR) was conducted according to a previous study (15).

Detection of m.T8993C mutation using PCR in combination with restriction fragment length polymorphism (PCR-RFLP) and sequencing. Total DNA from peripheral blood samples was manually extracted using the QIAamp DNA Mini kit (Qiagen, Valencia, CA, USA) and spectrophotometrically quantified by NanoDrop ND-1000 (NanoDrop; Thermo Fisher Scientific, Inc., Wilmington, DE, USA).

The m.T8993C mutation was detected using PCR-RFLP as previously described (16). Briefly, a mtDNA fragment of 402 bp length (spanning the 8768-9169 bp region of the mitochondrial genome) was amplified with the following primers. Forward primer: T8993C Fw 5'-CAACTAACCTCCTCG GAC-3' (corresponding to the $8768-8785$ bp region); reverse primer: T8993C Rv 5'-TGAAAACGTAGGCTTGGAT-3' (9169-9151 bp region). Amplified PCR products were digested using endonuclease HpaII, an enzyme that specifically recognizes and cuts after the first nucleotide in the $\mathrm{C} / \mathrm{CGG}$ sequence at the base pair 8,993 , and separated by $12 \%$ polyacrylamide gel electrophoresis. Ethidium bromide-stained gels were then photographed using Geldoc (Bio-Rad Laboratories, Inc., Hercules, CA, USA). The amplified and digested mtDNA of affected individuals showed three bands of lengths 402, 224, and $178 \mathrm{bp}$ (heteroplasmic), or two bands of lengths 224 and 178 bp (homoplasmic) in samples taken from affected individuals, whereas the mtDNA of normal of unaffected individuals showed only one band of length $402 \mathrm{bp}$. The presence of the m.T8993C mutant was reconfirmed using DNA sequencing (analyzed by the 1st Base Company, Singapore). The analyzed DNA sequence was then compared to the reference sequence (GenBank J01415.2).

Quantification of mtDNA copy number using $q P C R$. The m.T8993C heteroplasmy level was quantitated by qPCR using a Taqman Locked Nucleic Acid (LNA) probe (IDT Inc., Coralville,IA, USA) and iQ5 cycler (Biorad, USA) as previously described for the $\mathrm{A} 3243 \mathrm{G}$ mutation (17). In this case, a mtDNA fragment of 69 bp (8958-9026 bp region) was amplified with the following primers. Forward primer: RT8993-Fw 5'-CGA AACCATCAGCCTACTCATTCAA-3'; reverse primer: RT8993-Rv 5'-CCTGCAGTAATGTTAGCGGTTAGG-3' (corresponding to the 9026-9003 bp region). The LNA probes, Wt-8993T-HEX5'-/5HEX/AAT+AGCCC+T+GGCC/3IABkFQ-3' (8985-8997 bp region) and Mt-8993C-FAM 5'-/5FAM/ ATAGCCC+CGGCCGT/3IABkFQ/-3' (8986-8999 bp region), containing LNA nucleotides (nucleotides proceeded by the ' + ' symbol in the given sequences) were designed for quantitating the wild type and mutant genotypes, respectively. The copy numbers of mutant and wild-type mtDNA in the samples were calculated from standard curve, obtained by plotting the $\mathrm{C}_{\mathrm{t}}$ values of six standards on the $\mathrm{X}$-axis vs. the logarithm of their copy numbers on the Y-axis. The standards were 1:1 mixtures of the pJET1.2 plasmid with either the mutant m.T8993C or wild-type (T8993) DNA, at concentrations increasing from $5.10^{3}$ to $5.10^{6}$ copies per $\mu 1$ The calculations were made by averaging triplicate measurements. The level of heteroplasmy was defined as the percentage of mutant copies obtained in total copies of mutant and wild type DNA.

Statistical analysis. Data are presented as means \pm standard error of the mean (SEM). Differences between the generations were evaluated by Pair sample t-test using Origin 8.0 (version 8.0; OriginLab, Northampton, MA, USA). P<0.05 was considered to indicate a statistically significant difference.

Clinical features and MRI of the Leigh syndrome patient. MRI data of the patient revealed abnormal anatomical characteristics. Axial fluid-attenuated inversion recovery MRI (Axial FLAIR MR) showed a symmetric abnormal signal in the dorsal medulla oblongata. Axial T2 weighted image (T2WI) MRI revealed Sylvian fissure enlargement in association with abnormal signal in the periventricular white matter and in the putamina, and caudate heads (Fig. 1). The patient first presented with characteristics of Leigh syndrome at 21 months of age: muscular weakness (hypotonia), movement disorder, fever, intractable nausea and vomiting. His blood test results indicated lactatemia $(8.2 \mathrm{mmol} / 1 \mathrm{compared}$ to the normal level of less than $2.8 \mathrm{mmol} / \mathrm{l}$ ) and acidosis associated 
A a-1

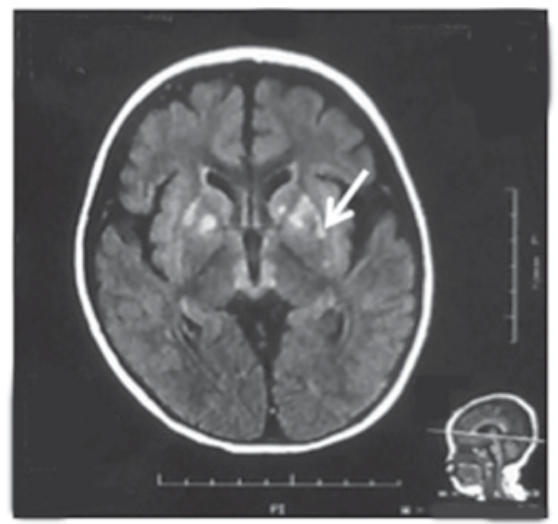

B b-1

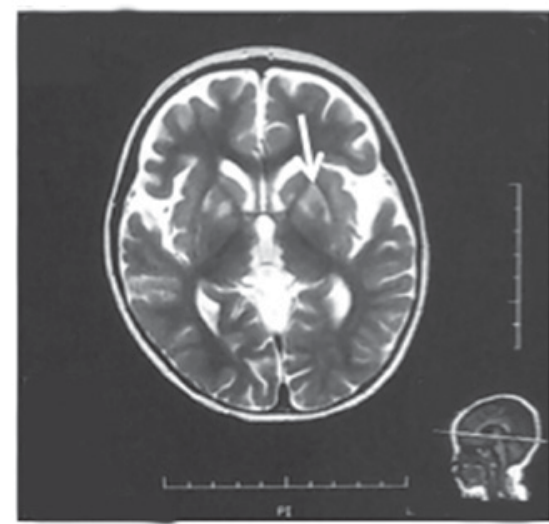

a-2

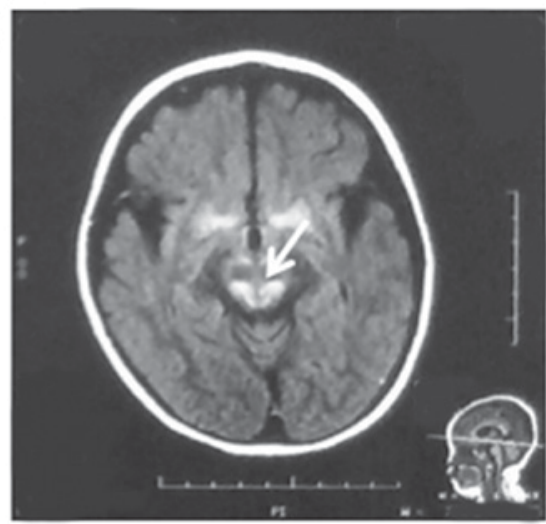

b-2

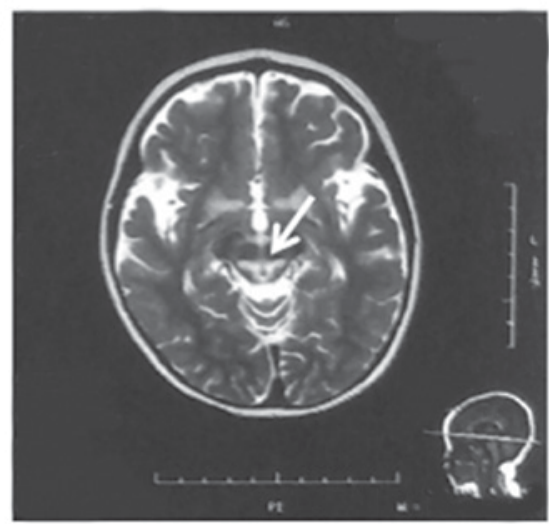

Figure 1. Magnetic resonance images of the brain representing typical Leigh syndrome characteristics in the brain of the patient. (A) Axial fluid-attenuated inversion recovery (FLAIR) image through the basal ganglia shows symmetrical hyperintense lesions involving the caudate nuclei, lentiform nuclei, medial thalami (a-1), and FLAIR image through the brainstem also shows symmetrical involvement of the cerebral peduncles and periaqueductal gray matter (a-2). (B) Axial T2 weighted image (T2WI) through the basal ganglia shows symmetrical hyperintense lesions involving the caudate nuclei, lentiform nuclei, medial thalami (b-1), and T2WI through the brainstem also shows symmetrical involvement of the cerebral peduncles and periaqueductal gray matter (b-2). White arrows indicate the damaged areas.

with ketonuria. During treatment, the patient showed severe cognitive retardation, episodes of seizures, pneumonia, lethargy, and fever. Severe hypotonia associated with dystonia and dyspnea was also found. The patient died at 34 months of age because of respiratory failure.

Discovery of m.T8993C point mutation in the patient and his family members. As $\mathrm{T}>\mathrm{C} / \mathrm{G}$ nucleotide changes at site 8,993 bp are commonly diagnosed in Leigh syndrome patients, we carried out PCR-RFLP using HpaII enzyme on the DNA samples. PCR-RFLP results showed that three DNA bands (402, 224, and $178 \mathrm{bp}$ ) were present in lanes 1, 2, 4-6, and 8 corresponding to DNA samples taken from the patient, his mother, his cousin 1 (male), his cousin 2 (female), his aunt, and his grandmother, as shown in Fig. 2. This indicates that these family members carried the m.T8993C/G point mutation and that the variant was maternally inherited from the grandmother. The mutation was not found in DNA samples taken from the rest of the family members including the father, the husband of the aunt, and the grandfather of the patient (Fig. 2; lanes 3, 7, and 9) as indicated by the presence of a single band of length $402 \mathrm{bp}$.

The DNA sequence obtained from the patient sample was then compared to the GenBank reference sequence (J01415.2). The presence of the $\mathrm{T} 8993 \mathrm{C}$ mutation in the representative alignment between the sequence of patient and the reference sequence is shown in Fig. 3. Based on the DNA sequencing data, we can conclude that the patient, his brothers and mother have the T8993C mutation. Heterogeneity in the intensities of the 224 and 178 bp bands among the samples containing the T8993C mutation probably reflect different levels of heteroplasmy (Fig. 2).

Quantification of m.T8993C heteroplasmy levels in family members. As the level of heteroplasmy of m.T8993C is an important factor affecting the phenotype and severity of Leigh syndrome, we reconfirmed the presence of the mutation and quantified heteroplasmy levels among the patient's family members in triplicate by qPCR using LNA Taqman probes.

Amplification curves for the mutant genotype (FAM channel, Fig. 4A) appeared only in samples from the patient, his mother, cousin 1 , his cousin 2 , his aunt, and his grandmother, but not in those from the patient's father, his aunt's husband, or his grandfather, whereas the amplification curves for the wild-type genotype (HEX channel, Fig. 4B) appeared in all the samples. The calculated heteroplasmy levels of the patient and his family members (based on the standard curves) were significantly different between the generations (Fig. 4 and Table I). The percentages of heteroplasmy levels in peripheral blood samples taken from the patient, his mother, cousin 1, cousin 2, his aunt, and his grandmother were 94.00 $\pm 1.34,71.66 \pm 3.22,87.00 \pm 1.79$, $91.24 \pm 2.50,66.81 \pm 0.85$, and $16.33 \pm 1.67 \%$, respectively. The 


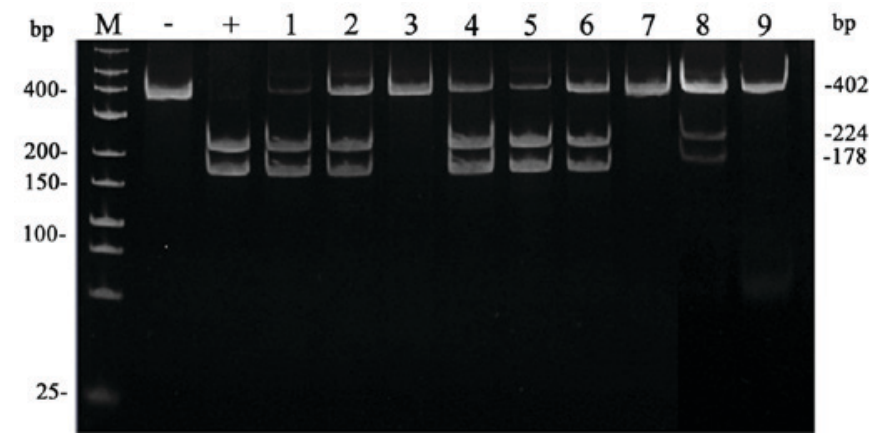

Figure 2. PCR-RFLP analysis of the m.T8993C mutation in the samples of the patient and his family members. Polyacrylamide electrophoresis results of $H p a I I$ enzyme digestions. Amplified mutant mtDNA shows bands of 402, 224, and $178 \mathrm{bp}$ (heteroplasmic) or bands of 402 and 224 bp (homoplasmic); wild-type mtDNA shows a band of $402 \mathrm{bp}$. Lane labels M, Marker (low range DNA ladder); -, Negative control; +, Positive control; 1, Patient; 2, Mother; 3 , Father; 4, Cousin 1 (male); 5, Cousin 2 (female); 6, Aunt; 7, Husband of the aunt; 8, Grandmother; 9 , Grandfather.

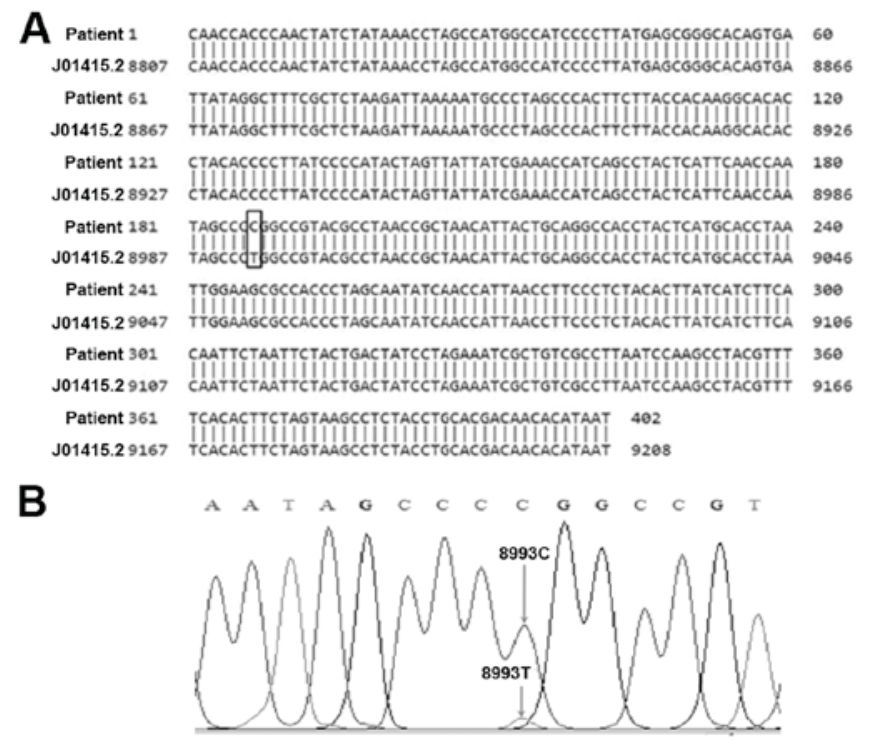

Figure 3. Nucleotide sequence of mtDNA from the patient carrying the T8993C mutation. (A) Alignment of mtDNA sequence encompassing the $8,807-9,167$ bp region of the patient with the reference sequence (GenBank J01415.2). (B) Sequencing chromatogram for nucleotide $T>C$ change at the $8,993 \mathrm{bp}$ site. The arrows denote the $\mathrm{T}>\mathrm{C}$ variation.

mutation was not detectable in samples from his grandfather, his father, or his aunt's husband.

The qPCR results presented in Fig. 4 and Table I also correlate well with the change from low to high intensity of the 224 and 178 bp bands obtained by PCR-RFLP (Fig. 2). The results reconfirmed that among mutation bearers, the grandmother harbored the level of heteroplasmy $(16.33 \pm 1.67 \%)$, while the patient bore the level of heteroplasmy $(94.00 \pm 1.34 \%)$. Both of his cousins harbored high levels of heteroplasmy, while his mother and his aunt bore milder levels of mutation loads.

\section{Discussion}

Leigh syndrome is a rare, heritable, heterogeneous, and progressive neurodegenerative disorder, appearing mostly during
Table I. Correlation between percentage m.T8993C mutation heteroplasmy and clinical phenotype in patient's family.

\begin{tabular}{lccc}
\hline $\begin{array}{l}\text { Family } \\
\text { members }\end{array}$ & $\begin{array}{c}\text { Age } \\
\text { (years) }\end{array}$ & $\begin{array}{c}\text { Clinical presence } \\
\text { of Leigh syndrome }\end{array}$ & $\begin{array}{c}\text { Mutation load } \\
(\%)\end{array}$ \\
\hline Patient & 2 & Yes & $94.00 \pm 1.34$ \\
Mother & 35 & No & $71.66 \pm 3.22$ \\
Father & 37 & No & $0.00 \pm 0.00$ \\
Cousin 1 & 6 & No & $87.00 \pm 1.79$ \\
Cousin 2 & 3 & No & $91.24 \pm 2.50$ \\
Aunt & 32 & No & $66.81 \pm 0.85$ \\
Aunt's husband & 34 & No & $0.00 \pm 0.00$ \\
Grandmother & 60 & No & $16.33 \pm 1.67$ \\
Grandfather & 62 & No & $0.00 \pm 0.00$ \\
\hline
\end{tabular}

infancy or early childhood $(1-3,7,18)$ and it affects approximately 1 in 40,000 live births worldwide (5). In Vietnam, no patients have been found in a previous screening (16). Here, we report the first case of a young Vietnamese patient clinically presenting the symptoms of Leigh syndrome who was genetically confirmed to harbor T8993C mtDNA mutation. In contrast to the patient, other patient family members carrying the T8993C mutation were neurologically asymptomatic and in them the percentage of heteroplasmy was lower than in the patient. The asymptomatic range was found to be between 16 and $91 \%$ approximately.

In this study, the patient presented clinical characteristics common to Leigh syndrome, as reported previously $(1,2,11,13,19)$. Clinical features of Leigh syndrome such as epilepsy, intractable nausea and vomiting accompanied by abnormal brain imaging were observed in the patient (Fig. 1). Similar to a previous study (2), the clinical features of our patient also included muscular hypotonia. The patient was also found to have lactatemia $(8.2 \mathrm{mmol} / \mathrm{l})$ and ketonuria. $\mathrm{He}$ lost the ability to speak and, eventually, could no longer walk at two years of age.

MRI of his brain revealed a symmetric abnormal signal in the dorsal medulla oblongata and Sylvian fissure enlargement in association with an abnormal signal in the periventricular white matter and in the putamina, and caudate heads (Fig. 1), which were pathognomonic for Leigh syndrome as demonstrated previously $(2,20)$. He also exhibited deterioration of cognitive and motor functions. In agreement with earlier reports (21), the patient was ultimately diagnosed with Leigh syndrome after targeted mutation analysis revealed high heteroplasmy (94\%) of mtDNA bearing the m.T8993C mutation. Although, m.T8993C heteroplasmy has also been associated with neurogenic weakness, ataxia and retinitis pigmentosa [NARP syndrome (22)], it's correlation with disease characteristics is not clearly understood (6), particularly for explaining the normal health of other family members of the patient. The patient was treated with coenzyme Q10 and vitamins and showed some progress. Unfortunately, he kept continuing to deteriorate and died of respiratory failure at 34 months of age during an episode of chest infection, as is commonly reported for such patients (14). 

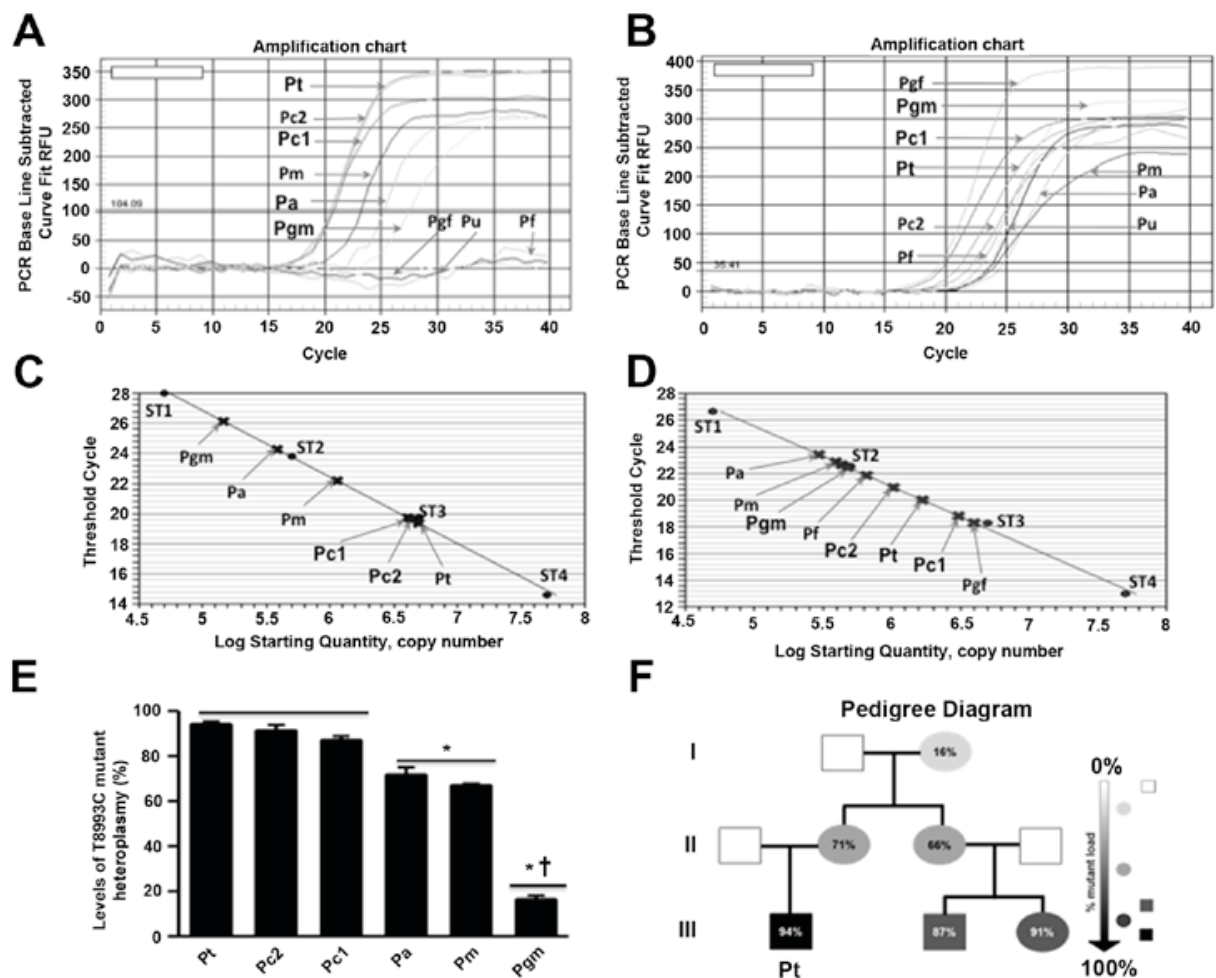

$\mathbf{F}$

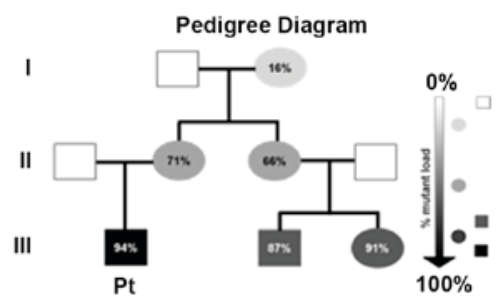

Figure 4. Quantitative polymerase chain reaction (qPCR) analysis of the T8993C mutation and pedigree diagram of the patient's family. (A) qPCR amplification curves quantifying the T8993C mutation in samples of the patient and his family members using the (A) FAM and (B) HEX LNA probes. Standard curves of Ct values generated by mixing plasmid DNA with varying concentrations of (C) $100 \%$ mutant or (D) $100 \%$ wild-type DNA, with overlaid Ct values of the patient and his family members. ST1, ST2, ST3, and ST4 are the concentrations of standards $\left(5.10^{3}, 5.10^{4}, 5.10^{5}\right.$ and $5.10^{6}$ molecules/ $\mu 1$, respectively). (E) Heteroplasmy levels (\%) in family members harboring the m.T8993C mutation. ${ }^{*} \mathrm{P}<0.05$ vs. the 3 rd generation, ${ }^{\dagger} \mathrm{P}<0.05$ vs. the $2 \mathrm{nd}$ generation. (F) Pedigree diagram of the family. The gray or black symbols show affected family members and the white symbol indicates unaffected members. Numbers refer to percentage of mutated mtDNA in the blood of family members. Circles and squares denote females and males, respectively. Pt, Patient; Pm, Patient's mother; Pf, Patient's father; Pc1, Patient's cousin 1; Pc2, Patient's cousin 2; Pa, Patient's aunt; Pu, The husband of patient's aunt; Pgm, Patient's grandmother; Pgf, Patient's grandfather.

Mutation at the mtDNA nucleotide 8993 site is a relatively common cause of Leigh syndrome (8) with a correlation between the amount of mutant mtDNA and clinical severity $(11,20,22)$. The change of nucleotide (either $\mathrm{T}>\mathrm{G}$ or $\mathrm{T}>\mathrm{C}$ ) at this site is hypothesized to impair OXPHOS, particularly I and V complexes, leading to ROS over-generation and mitochondrial energy metabolic malfunction, with subsequent cell death (11). It is reported that the mtDNA 8993 mutation site is in heteroplasmy, with cells having both wild-type and mutant mtDNA and the heteroplasmy level is an important factor determining the phenotype and severity of the disease (12). The tissues demanding high energy are most vulnerable to this process and, in part, are responsible for the clinical characteristics of Leigh syndrome patients. The mtDNA T8993C mutation is generally considered to be clinically milder than the T8993G mutation (11). However, in cases where the levels of heteroplasmy exceed approximately $90-95 \%$, progressive neurodegeneration characterized by bilateral lesions of the basal ganglia, which may extend to the brainstem, lead to the eventual death of the patient $(13,22)$. In our study, the patient's second cousin carried the T8993C mutation at $91.24 \pm 2.50 \%$ levels of heteroplasmy but showed no clinical symptoms of Leigh syndrome. This level of heteroplasmy is still very close to the normal range of reported cases $(11,22)$; even a case of $\geq 95 \%$ of heteroplasmy has been reported not to show most symptoms of Leigh syndrome (22). In concordance with the literature $(11,22)$, we found that our Vietnamese patient presenting with clinical
Leigh syndrome harbored the highest heteroplasmy level of the T8993C mutation in his family. The other family members carrying T8993C mutation were neurologically asymptomatic, with lower percentages of heteroplasmy compared to that of the patient (Fig. 4 and Table I). Prenatal diagnosis of the T8993C mutation is technically possible and there is a correlation between the percentage of heteroplasmy of the T8993C mutation and the clinical phenotype (12). Similar to a previous report regarding Leigh patients harboring high T8993C heteroplasmy levels in the blood (10), our patient showed worsening clinical progression, was unable to speak, hypotonic, and also showed motor disability. However, variability in the phenotypic expression of patients carrying the T8993C mutation has also been observed $(3,11,22)$. As the level of heteroplasmy of mtDNA in the patient's blood was at approximately $95 \%$, he showed early muscular hypotonia and abnormal brain MRI (11). Another case report described the long-term follow-up of a Leigh syndrome patient who presented at four years of age with the typical T8993C mutation (95\% heteroplasmy), but showed an unexpected resolution of symptoms and a favorable outcome. This suggests the need for caution in predictive counseling of patients suspected to have Leigh syndrome and their parents (22). No strong correlation between the clinical features and heteroplasmy has been reported (3). Therefore, co-variation in heteroplasmy among tissues and/or over time, and the clinical presentation of Leigh syndrome with brainstem or spinal cord involvement should 
be tracked in patients with this syndrome $(6,14)$. In our study, except for typical clinical presentation by the patient, all the family members carrying the mutation were clinically normal. A pedigree analysis confirmed maternal inheritance of the T8993C mutation with heteroplasmy. Genetic testing revealed that the proportion of the mtDNA carrying the T8993C mutation was $16.33 \pm 1.67$ in the patient's maternal grandmother, $71.66 \pm 3.22 \%$ in the patient's mother, and $66.81 \pm 0.85 \%$ in the patient's maternal aunt. The grandmother, mother, and the aunt were all clinically normal. The patient also had two symptomatic maternal first cousins, the aunt's children, who had approximately 87 to $91 \%$ mtDNA with the m.T8993C mutation, but they were neurologically asymptomatic. This finding can be explained by the tight correlation between the heteroplasmy and the development of the phenotype in T8993C carriers $(11,23)$. That is, the threshold of heteroplasmy of this mutation for the appearance of effects is high. Moreover, the mutant load can change over time and the clinical characteristics of individuals could be dependent on other factors, such as the frequency of environmental stressors like illness $(6,9)$. As mentioned above, nucleotide change at mtDNA 8,993 bp affects ATPase activity and thus be associated with MILS or NARP when the mutational load is greater than $90 \%$, or between $50-60 \%$, respectively $(13,22)$. Greater than $95 \%$ proportion of m.T8993C mutation has also been revealed in an adult diagnosed with NARP syndrome $(19,22)$.

The varying degrees of heteroplasmy produce a wide range of clinical symptoms and most T8993C carrying patients present one or more abnormal neurologic characteristics, including brainstem or basal ganglia dysfunction, seizures, hypotonia, periodic illness, and progressive neurodegeneration $(2,19,23)$. As a bioenergetic defect is unlikely to be the main reason for explaining all the Leigh syndrome-associated phenotypes and their pathogenesis $(13,24)$, more study is required, especially to address the higher frequency of ataxia in T8993C-based Leigh syndrome cases (23). Predictably, ATP synthesis rate was only mildly reduced by the m.T8993C mutation at levels of $94 \%$ heteroplasmy (13), especially because only a $22 \%$ reduction of ATP synthesis has been previously reported in homoplasmic m.T8993C cybrids (24). So far, increased oxidative stress may be the major cause of the occurrences of MILS cases associated with the T8993C mutation (13). At the molecular level, the difference in severity between T8993C and T8993G carrying patients is that the T8993C mutation results in substitution of leucine with proline (p.Leu156Pro) in ATP synthase subunit 6 (Atp6p), while the T8993G mutation results in substitution of leucine with arginine (p.Leu156Arg) in Atp6p (11). Leu and Pro both are hydrophobic amino acids and their side residues have similar sizes. Thus the Leu vs. Pro changes should not be very different with respect to their effect on activity of Atp6p protein. However, replacement of Leu by Arg does induce a structural defect in human $\mathrm{F}_{1} \mathrm{~F}_{0}$-ATPase that causes a severe impairment in ATP synthesis (25).

It is well known that mtDNA mutations are randomly subjected to a bottleneck effect and segregate to the oocytes forming in the female fetus (26). Differential amplification could lead to a rapid diversification of mtDNA genotypes in a single generation. Heteroplasmy and mutant types (either m.T8993G or m.T8993C) play important roles in determining the age of disease onset as well as in levels of clinical severity.
As pointed out in Fig. 4 and Table I, the heteroplasmic state of the m.T8993C mutation was significantly increased over successive generations in this Vietnamese family. Interestingly, in the two preceding generations, none of the paternal members harbor the mutation; only mothers and their children carry the variant (as seen in the pedigree diagram in Fig. 4F). Perhaps, it is a result of a bottleneck effect $(9,24)$. If this effect takes place during or after early embryonic development, there may be variable mutational loads in tissues and between ages. We suppose that the high heteroplasmic presentation in our patient was probably due to random segregation and that a bottleneck effect occurred during his mother's oogenesis. In contrast to this patient, the mtDNA genetic bottleneck effect and genetic drift in other family members might have occurred during gastrulation because their genetic profiles did not match their clinical records (27). However, severity of Leigh syndrome occasionally depends on the tissue distribution and on the mutant load of the pathogenic variant $(6,24)$. In this family, all children of females with the mtDNA pathogenic variant remained at risk of developing Leigh symptoms. In agreement with the previous studies about this disease, mothers had much lower mutation loads than children and displayed normal health (9), suggesting that the m.T8993C mutation is likely to have a higher tendency of being selected for during early oogenesis. Here, the patient was found to have the highest heteroplasmy of m.T8993C with typical clinical features of Leigh syndrome while other family member remained asymptomatic. In the first generation, the patient's grandmother bore the lowest level of mutational load (approximately 16\%). His mother and aunt, the members of the second generation, exhibited a lower heteroplasmy percentage (between 67 and 71\%). The patient and his two younger cousins, representative of the third generation, harbored the highest mutational burden (approximately 91\%). it is possible that the T8993C mutation could aggregate rapidly towards homoplasmy through generations of this family, and their clinical manifestations become more severe, as has been demonstrated in other cases (9).

To date, there is no cure for mtDNA T8993C mutation-affected patients, and treatment options are mostly unsatisfactory (18). Furthermore, mitochondrial genetics, ATP production, ROS levels, and other undefined factors likely differentiate the onset and severity of the disorder (19). In order to understand correspondence between the genetic profiles and clinical manifestation more investigation is needed.

In conclusion, this is the first case of an m.T8993C mutation-associated Leigh syndrome case identified in Vietnam. We also described the correlation between the percentage of heteroplasmy of this mutation and the clinical phenotype of the patient's family. We hope that these data lead to the improvement of clinical diagnosis and treatment of Leigh syndrome patients.

\section{Acknowledgements}

Not applicable.

\section{Funding}

The present study was supported by the Vietnam National University, Hanoi (Project code: KLEPT.16.03 to TNP). 


\section{Availability of data and materials}

The datasets used and/or analyzed during the current study are available from the corresponding author on reasonable request.

\section{Authors' contributions}

CALW performed qPCR analysis of the patient and his family members, BHTB performed PCR-RFLP analysis of the patient and his family, TTV isolated DNA from the samples and contributed to writing the manuscript, HLTN analyzed qPCR data, prepared the figures and contributed to writing the manuscript, BKP checked the PCR-RFLP data, VMN checked the $\mathrm{qPCR}$ data, VAP contributed to assessing the clinical data of the patient and his family members, VHC analyzed NMR data, diagnosed and treated the patient and contributed to writing the manuscript. TNP coordinated the study with TTV, HLTN and VHC and finalized the manuscript. All authors read and approved the final manuscript.

\section{Ethics approval and consent to participate}

The present study was approved by the Ethics Committee of the $\mathrm{VNCH}$ and the patient's representative and his family members signed consent forms to participate in the study.

\section{Consent for publication}

The authors declare that identifying information, including names, initials, date of birth or hospital numbers, images of the patient and his family members were not included in the manuscript and publication of the data presented in the manuscript does not compromise anonymity or confidentiality.

\section{Competing interests}

The authors declare that they have no competing interests.

\section{References}

1. Leigh PN, Al-Sarraj S and DiMauro S: Impact commentaries. Subacute necrotising encephalomyelopathy (Leigh's disease; Leigh syndrome). J Neurol Neurosurg Psychiatry 86: 363-365, 2015.

2. Piao YS, Tang GC, Yang H and Lu DH: Clinico-neuropathological study of a Chinese case of familial adult Leigh syndrome. Neuropathology 26: 218-221, 2006.

3. Finsterer J: Leigh and leigh-like syndrome in children and adults. Pediatr Neurol 39: 223-235, 2008.

4. Thorburn DR, Rahman J and Rahman S: Mitochondrial DNA-associated Leigh syndrome and NARP. In: Adam MP, Ardinger HH, Pagon RA, Wallace SE, Bean LJH, Stephens K, Amemiya A, editors. Source GeneReviews ${ }^{\circledR}$ [Internet]. University of Washington, Seattle, WA, 1993-2018.

5. Piekutowska-Abramczuk D: The molecular background of Leigh syndrome. Neurol Neurochir Pol 42: 238-250, 2008 (In Polish).

6. White SL, Shanske S, McGill JJ, Mountain H, Geraghty MT, DiMauro S, Dahl HH and Thorburn DR: Mitochondrial DNA mutations at nucleotide 8993 show a lack of tissue- or age-related variation. J Inherit Metab Dis 22: 899-914, 1999.

7. Bonfante E, Koenig MK, Adejumo RB, Perinjelil V and Riascos RF: The neuroimaging of Leigh syndrome: Case series and review of the literature. Pediatr Radiol 46: 443-451, 2016.

8. Santorelli FM, Shanske S, Macaya A, DeVivo DC and DiMauro S: The mutation at nt 8993 of mitochondrial DNA is a common cause of Leigh's syndrome. Ann Neurol 34: 827-834, 1993.
9. Makino M, Horai S, Goto Y and Nonaka I: Mitochondrial DNA mutations in Leigh syndrome and their phylogenetic implications. J Hum Genet 45: 69-75, 2000.

10. Santorelli FM, Shanske S, Jain KD, Tick D, Schon EA and DiMauro S: A T->C mutation at nt 8993 of mitochondrial DNA in a child with Leigh syndrome. Neurology 44: 972-974, 1994.

11. Morava E, Rodenburg RJ, Hol F, de Vries M, Janssen A, van den Heuvel L, Nijtmans L and Smeitink J: Clinical and biochemical characteristics in patients with a high mutant load of the mitochondrial T8993G/C mutations. Am J Med Genet A 140A: 863-868, 2006.

12. Tatuch Y, Pagon RA, Vlcek B, Roberts R, Korson M and Robinson BH: The 8993 mtDNA mutation: Heteroplasmy and clinical presentation in three families. Eur J Hum Genet 2: 35-43, 1994.

13. Baracca A, Sgarbi G, Mattiazzi M, Casalena G, Pagnotta E, Valentino ML, Moggio M, Lenaz G, Carelli V and Solaini G: Biochemical phenotypes associated with the mitochondrial ATP6 gene mutations at nt8993. Biochim Biophys Acta 1767: 913-919, 2007.

14. Huntsman RJ, Sinclair DB, Bhargava R and Chan A: Atypical presentations of Leigh syndrome: A case series and review. Pediatr Radiol 32: 334-340, 2004.

15. Bakshi R, Ariyaratana S, Benedict RH and Jacobs L: Fluid-attenuated inversion recovery magnetic resonance imaging detects cortical and juxtacortical multiple sclerosis lesions. Arch Neurol 58: 742-748, 2001.

16. Truong HT, Nguyen TVA, Nguyen LV, Pham VA and Phan TN: Screening of common point-mutations and discovery of new T14727C change in mitochondrial genome of Vietnamese encephalomyopathy patients. Mitochondrial DNA A DNA Mapp Seq Anal 27: 441-448, 2016.

17. Truong HT, Nguyen TVA, Nguyen THL, Pham VA and Phan TN: Sensitive quantification of mitochondrial mutation using new Taqman probes. Cent Eur J Med 9: 839-848, 2014.

18. Kumagai R, Ichikawa K, Yasui T, Kageyama Y and Miyabayashi S: Adult Leigh syndrome: Treatment with intravenous soybean oil for acute central respiratory failure. Eur J Neurol 6: 613-615, 1999.

19. Ruhoy IS and Saneto RP: The genetics of Leigh syndrome and its implications for clinical practice and risk management. Appl Clin Genet 7: 221-234, 2014.

20. Makino M, Horai S, Goto Y and Nonaka I: Confirmation that a T-to-C mutation at 9176 in mitochondrial DNA is an additional candidate mutation for Leigh's syndrome. Neuromuscul Disord 8: 149-151, 1998.

21. Enns GM, Bai RK, Beck AE and Wong LJ: Clinical correlations in a family with variable tissue mitochondrial DNA T8993G mutant load. Mol Genet Metab 88: 364-371, 2006.

22. Debray FG, Lambert M, Lortie A, Vanasse M and Mitchell GA: Long-term outcome of Leigh syndrome caused by the NARP-T8993C mtDNA mutation. Am J Med Genet A 143A: 2046-2051, 2007.

23. Fujii T, Hattori H, Higuchi Y and Tsuji M: Phenotypic differences between $\mathrm{T}->\mathrm{C}$ and $\mathrm{T}->\mathrm{G}$ mutations at nt 8993 of mitochondrial DNA in Leigh syndrome. Pediatr Neurol 18: 275-277, 1998.

24. Pallotti F, Baracca A, Hernandez-Rosa E, Walker WF, Solaini G, Lenaz G, Melzi D'Eril GV, Dimauro S, Schon EA and Davidson MM: Biochemical analysis of respiratory function in cybrid cell lines harbouring mitochondrial DNA mutations. Biochem J 384: 287-293, 2004.

25. Baracca A, Barogi S, Carelli V, Lenaz G and Solaini G: Catalytic activities of mitochondrial ATP synthase in patients with mitochondrial DNA T8993G mutation in the ATPase 6 gene encoding subunit a. J Biol Chem 275: 4177-4182, 2000.

26. Dahl HH, Thorburn DR and White SL: Towards reliable prenatal diagnosis of mtDNA point mutations: Studies of nt8993 mutations in oocytes, fetal tissues, children and adults. Hum Reprod 15 (Suppl 2): S246-S255, 2000.

27. St John JC, Facucho-Oliveira J, Jiang Y, Kelly R and Salah R: Mitochondrial DNA transmission, replication and inheritance: A journey from the gamete through the embryo and into offspring and embryonic stem cells. Hum Reprod Update 16: 488-509, 2010. 